\title{
Tuning insulator-semimetal transitions in 3D topological insulator thin films by inter-surface hybridization and in-plane magnetic fields
}

\author{
Yang Xu, ${ }^{1,2}$ Guodong Jiang, ${ }^{1}$ Ireneusz Miotkowski, ${ }^{1}$ Rudro R. Biswas, ${ }^{1,3}$ and Yong P. Chen ${ }^{1,2,3,4,5}$, 故 \\ ${ }^{1}$ Department of Physics and Astronomy, Purdue University, West Lafayette, IN 47907, USA \\ ${ }^{2}$ Birck Nanotechnology Center, Purdue University, West Lafayette, IN 47907, USA \\ ${ }^{3}$ Purdue Quantum Center, Purdue University, West Lafayette, IN 47907, USA \\ ${ }^{4}$ School of Electrical and Computer Engineering, \\ Purdue University, West Lafayette, IN 47907, USA \\ ${ }^{5}$ WPI-AIMR International Research Center on Materials Sciences, Tohoku University, Sendai, 980-8577 Japan
}

(Dated: April 9, 2019)

\begin{abstract}
A pair of Dirac points (analogous to a vortex-antivortex pair) associated with opposite topological numbers (with $\pm \pi$ Berry phases) can be merged together through parameter tuning and annihilated to gap the Dirac spectrum, offering a canonical example of a topological phase transition. Here, we report transport studies on thin films of BiSbTeSe $_{2}$ (BSTS), which is a 3D TI that hosts spinhelical gapless (semi-metallic) Dirac fermion surface states (SS) for sufficiently thick samples, with an observed resistivity close to $h / 4 e^{2}$ at the charge neutral point. When the sample thickness is reduced to $\sim 10 \mathrm{~nm}$ thick, the Dirac cones from the top and bottom surfaces can hybridize (analogous to a "merging" in the real space) and become gapped to give a trivial insulator. Furthermore, we observe that an in-plane magnetic field can drive the system again towards a metallic behavior, with a prominent negative magnetoresistance (MR, up to $\sim-95 \%$ ) and a temperature-insensitive resistivity close to $h / 2 e^{2}$ at the charge neutral point. The observation is interpreted in terms of a predicted effect of an in-plane magnetic field to reduce the hybridization gap (which, if small enough, may be smeared by disorder and a metallic behavior). A sufficiently strong magnetic field is predicted to restore and split again the Dirac points in the momentum space, inducing a distinct 2D topological semimetal (TSM) phase with 2 single-fold Dirac cones of opposite spin-momentum windings.
\end{abstract}

A wide range of quantum materials including graphene, topological insulators (TIs), Dirac/Weyl semimetals, and their artificial analogues, have been identified whose low-energy excitations behave as massless Dirac particles to host novel relativistic quantum phenomena [1] 7]. The Dirac spectra can be gapped by breaking the underlying symmetry that protects the Dirac points (DPs), or by pairwise merging and annihilation of DPs 6-12. Previously predicted material platforms to explore this latter mechanism, such as graphene with engineered anisotropic nearest-neighbor hopping 9 and thin black phosphorus under a strong electric field [10, require extreme parameter tuning that is difficult to realize experimentally [11-13]. Alternative platforms that have enabled experimental demonstration of this effect include a microwave analogue of strained graphene [6] and cold atoms in honeycomb optical lattices [7. On the other hand, 3D TI thin films with hybridization gapped surface states bring new opportunities to study such topological transitions in a solid-state system. In particular, merging and annihilating of top and bottom surface DPs (with opposite spin windings) can be controlled both in the real space (by sample thickness, for example demonstrated experimentally in Ref. [14 by angle-resolved photoemission spectroscopy on thin films grown by molecular beam epitaxy) and the momentum space (by an in-plane magnetic field, as theoretically proposed in Ref. [15]).

In a relatively thick 3D TI film (thickness $t \gg 10 \mathrm{~nm}$ ), the top and bottom surfaces are well separated and their corresponding topological SS Dirac cones are gapless with opposite spin helicities. When the sample is thin enough (typically $\leq \sim 10 \mathrm{~nm}$ ) to enable hybridization between the two surfaces, a gap $\Delta_{0}$ is opened at the DP (even though the time-reversal symmetry is still preserved). The SS band structure acquires massive Dirac dispersion $\varepsilon= \pm \sqrt{\left(\hbar v_{f} k\right)^{2}+\left(\Delta_{0} / 2\right)^{2}}$, with $\hbar$ being the Plank constant $h$ divided by $2 \pi, v_{f}$ the Fermi velocity and $k$ the (in-plane) wave vector 14. Such a crossover of 3D TIs to the two-dimensional (2D) limit, as well as their response to magnetic fields, is little explored by electronic transport measurements in 3D TI materials with no bulk conduction and surface-dominant transport (such as BSTS). Previous in-plane magneto-transport studies in 3D TIs often suffer from their residual bulk conduction [16, 17] and few have been reported in the hybridization regime [18 20].

Our experiment is based on a 3D TI crystal BSTS $\left(\mathrm{BiSbTeSe}_{2}\right)$ that has no detectable bulk conducting carriers at low temperature, with DPs of the topological SS exposed in the bulk band gap [21, 22, thus ideal for the study of low energy excitations in the vicinity of the surface DPs. The dual-gated BSTS devices 22 were fabricated into Hall-bar structures (with channel length $l$, width $w$, thickness $t$ ) on highly p-doped $\mathrm{Si}$ substrates (with $300 \mathrm{~nm}$-thick $\mathrm{SiO}_{2}$ coating). Hexagonal boron nitride (h-BN) flakes (tens of $\mathrm{nm}$ in thickness) are transferred onto the devices as top-gate dielectrics (see a typ- 

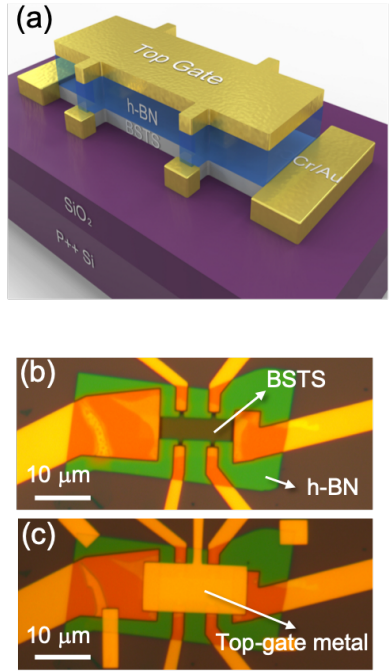

(d)

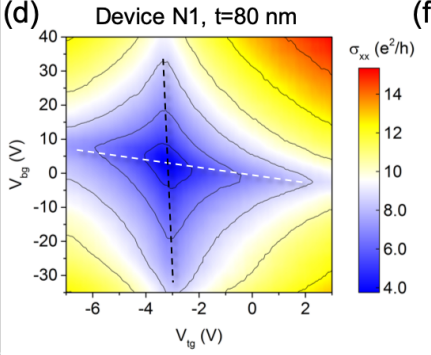

(e)

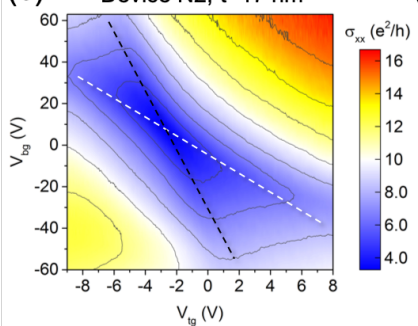

(f)

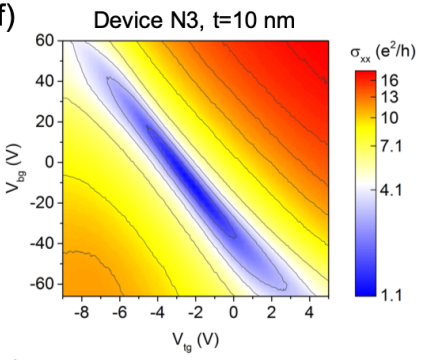

(g)

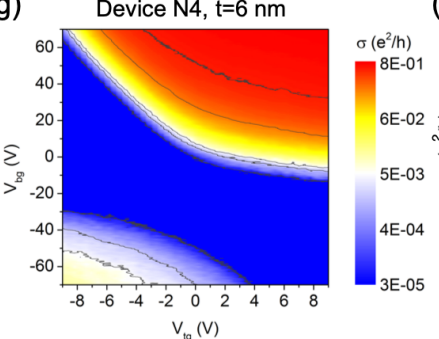

(h)

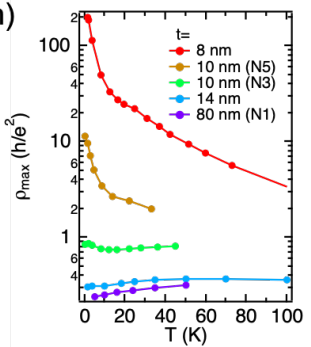

(i)

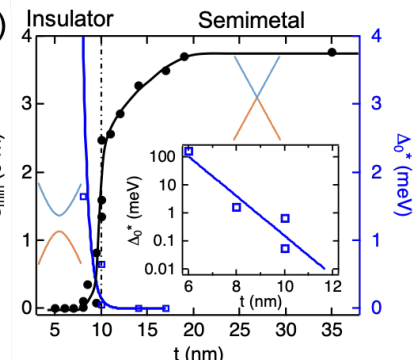

FIG. 1. (a) Schematic of a dual-gated TI device based on BSTS. (b,c) Optical images of Device N3 before (b) and after (c) top-gate metal deposition. (d-g) Measured conductivities (in color scale, with contours) as functions of $V_{t g}$ and $V_{b g}$ in four representative devices with decreasing thickness $(t)$. In $(\mathrm{d}, \mathrm{e})$ the black/white dashed lines trace the top/bottom surface DPs. Data are measured at temperature $T=0.35 \mathrm{~K}$ in $(\mathrm{d}-\mathrm{f})$, and at $T=1.6 \mathrm{~K}$ in $(\mathrm{g})$. (h) Temperature dependence of $\rho_{\max }(\log$ scale) for 5 representative devices. (i) The $\sigma_{\min }\left(=1 / \rho_{\max }\right.$, left axis) at low $T(<2 \mathrm{~K})$ and the extracted gap $\Delta_{0}^{*}$ (right axis) as functions of sample thickness $t$. The dashed-dotted vertical line marks the critical thickness $t_{c}=\sim 10 \mathrm{~nm}$ that separates the semimetal $\left(t>t_{c}\right.$, corresponding to the 3D TI phase in the inset with gapless Dirac SS) and insulator $\left(t \leq t_{c}\right.$, corresponding to the trivial insulator phase in the inset with gapped SS) behaviors. The inset plot shows $\Delta_{0}^{*}$ in log scale versus $t$ and an exponential fitting.

ical device schematic in Fig. 1a and optical images of Device N3 in Fig. 1b, c). Top and back gate voltages (denoted as $V_{t g}$ and $V_{b g}$ ) relative to the BSTS flake are applied to the top-gate metal and the doped $\mathrm{Si}$, respectively. Upon dual-gating, the carrier types and densities of both the top and bottom surfaces, thus the measured conductivity, can be modulated. By reducing the thickness of the BSTS flake, the capacitive coupling between the two surfaces becomes stronger [23, 24]. As it can be seen in the color map of $2 \mathrm{D}$ conductivity $\left(\sigma_{x x}=l /\left(w R_{x x}\right)\right.$, with $R_{x x}$ being the longitudinal resistance) versus $V_{t g}$ and $V_{b g}$ measured at low temperature, the black and white dashed lines tracing the DPs of top and bottom surfaces tend to merge together when the thickness $t$ is reduced from 80 $\mathrm{nm}$ to $17 \mathrm{~nm}$ (Fig. $1 \mathrm{~d}$ and 1e). Further reducing $t$ to $\sim 10$ $\mathrm{nm}$ results in the DPs from the two surfaces to become indistinguishable (Fig. 1f). When the sample is only a few nm thick (e.g., Device N4 with $t=6 \mathrm{~nm}$ in Fig. $1 \mathrm{~g}$ ), a hard gap opens, as indicated by the highly insulating (two-terminal conductivity $\sigma \ll e^{2} / h$ ) blue region.

The minimum conductivity $\sigma_{\min }$ and maximum resistivity $\rho_{\max }\left(=1 / \sigma_{\min }\right)$ are reached when the two surfaces are gated simultaneously to charge neutrality or DPs. In Fig. 1h, we plotted $\rho_{\max }$ as a function of temperature $(T)$ for a few representative samples. At $t>10 \mathrm{~nm}, \rho_{\max }$ shows a metallic behavior $\left(d \rho_{\max } / d T>0\right)$, implying a zero or negligible gap. However, at $t<10 \mathrm{~nm}$, a strong insulating behavior $\left(d \rho_{\max } / d T<0\right)$ is observed. Around $t=10 \mathrm{~nm}$, different samples can behave differently. For example, while device N5 exhibits an insulating behav- ior, another device N3 exhibits a non-monotonic temperature dependence with its $\rho_{\max }(T)$ close to $h / e^{2}$ and separating curves with metallic and insulating behaviors. It is consistent with the general observation from previous studies that the critical resistivity for metal-insulator transition in $2 \mathrm{D}$ electron systems is on the order of the resistance quantum $h / e^{2}$ [25]. Fig. 1i shows $\sigma_{\min }$ at base temperatures $(T \leq 1.6 \mathrm{~K})$ for samples with various thicknesses. At large $t(>\sim 20 \mathrm{~nm}), \sigma_{\min }$ saturates around a value close to $4 e^{2} / h 22$. The $\sigma_{\min }$ starts to decrease below $20 \mathrm{~nm}$ and drops abruptly to zero below $\sim 10 \mathrm{~nm}$. For samples that exhibits insulating behaviors, their $\rho_{\max }(T)$ were fitted to thermal activation behavior $\rho_{\max }(T) \propto e^{\Delta_{0}^{*} / 2 k_{B} T}$ (with $k_{B}$ being the Boltzmann constant) over appropriate temperature ranges to extract (see SI for details) the non-zero gap $\Delta_{0}^{*}$, plotted on the right axis of Fig. 1i. The $\Delta_{0}^{*}$ grows by about an order of magnitude when $t$ is reduced by $\sim 1.4 \mathrm{~nm}$ (see the exponential fitting in the inset of Fig. 1i), comparable to what was found for $\mathrm{Bi}_{2} \mathrm{Se}_{3}$ [14, 26]. Our data suggest that a measurable transport gap $\Delta_{0}^{*}$ (presumably driven by the inter-surface hybridization) opens at the DPs below a critical thickness $t_{c}=10 \pm 1 \mathrm{~nm}$ in our samples.

We have found that the resistances of the thicker and thinner samples respond to the in-plane magnetic field differently at low temperatures. For consistency, the samples are mounted with current direction parallel to $B$ (unless otherwise specified). We have measured multiple samples by either sweeping $V_{t g}$ (with $V_{b g}$ carefully tuned and then fixed at voltages such that these $V_{t g}$ 
(a)
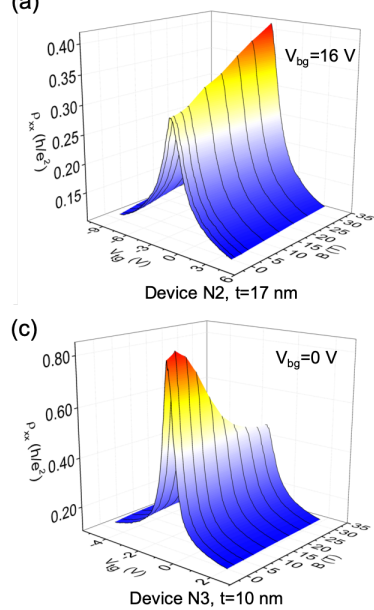

(e)

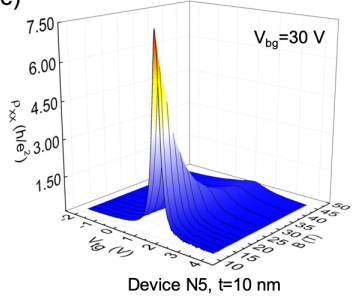

(b)

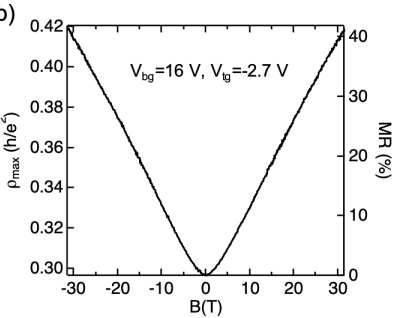

(d)

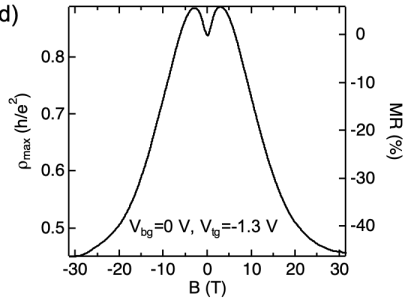

(f)

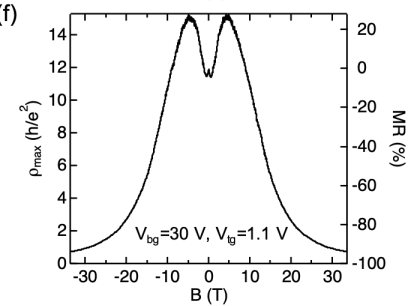

FIG. 2. (a,c,e) Resistivity of three representative devices (N2, N3 and N5) measured as a function of $V_{t g}$ at various in-plane $B$ fields at $T=0.3 \mathrm{~K}$. The corresponding $V_{b g}$ 's are carefully tuned such that the $V_{t g}$ sweeps go through $\rho_{\max }$. (b,d,f) The $\rho_{\max }$ (and the corresponding MR, right axis) measured (at fixed $V_{b g}$ and $V_{t g}$ labeled in the figure) as a function of in-plane $B$ field for the three devices (N2, N3 and N5 respectively).

sweeps go through $\rho_{\max }$ ) at different in-plane $B$ fields, or measuring $\rho_{\max }$ versus in-plane $B$ at fixed gate voltages. For relatively thick samples such as Device N2 with $t=17 \mathrm{~nm}>t_{c}$, the in-plane field up to $\sim 31 \mathrm{~T}$ only induced a relatively small positive MR of $\sim 40 \%$ (Fig. 2a and $2 \mathrm{~b}$, noting $\rho_{\max }(B)$ is approximately proportional to $B^{2}$ at low fields and to $B$ at higher fields). At low fields $(<\sim 5 \mathrm{~T}$ ), thinner devices N3 and N5 (both $\sim 10$ $\mathrm{nm}$ ) also show some positive MR (for N5, we also observed an additional tiny cusp with negative MR near 0 T). Such low-field features in thinner devices disappear when we increase the temperature to just a few Kelvin (see SI), thus are attributed to phase coherent transport [18, 19]. In the following, we mainly focus on the higher field data showing a giant negative MR that has only been observed in thin samples with insulating behavior (hybridization gapped). For example, in Device N3 (Fig. $2 \mathrm{c}$ and $2 \mathrm{~d}), \rho_{\max }$ drops dramatically above $\sim 5 \mathrm{~T}$ and saturates at high field $(\sim 30 \mathrm{~T})$ to $\sim 0.45 h / e^{2}$. Notably for the more insulating sample N5 (Fig. 2e and 2f), $\rho_{\max }$ drops by a factor of $\sim 20$ (giving an $\mathrm{MR} \sim-95 \%$ ) from a very resistive value of $\sim 12 h / e^{2}$ at $B=0 \mathrm{~T}$ to a value $\left(\sim 0.55 h / e^{2}\right)$ again close to $h / 2 e^{2}$ at $B=45 \mathrm{~T}$. We have verified that Device N5 also showed a large negative MR $(-85 \%$ at $31 \mathrm{~T})$ when the in-plane $B$ field is perpendicu-
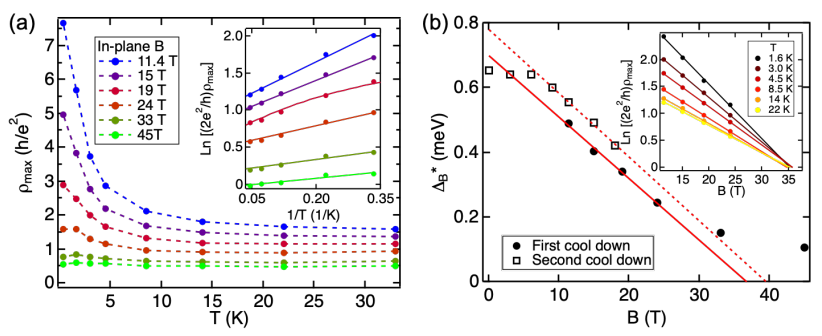

FIG. 3. (a) The $\rho_{\max }$ of device $\mathrm{N} 5(t \sim 10 \mathrm{~nm})$ vs. $T$ at different in-plane B from $11.4 \mathrm{~T}$ up to $45 \mathrm{~T}$. Inset shows corresponding thermal activation $\left(\rho_{\max }(T) \propto e^{\Delta_{B}^{*} / 2 k_{B} T}\right)$ fittings, while the extracted $\Delta_{B}^{*}$ is plotted (filled circles) as a function of $B$ in (b) along with data measured from another (second) cool down. (b) The linear fits for both cool downs indicate a gap closing at $B_{c}$ between 35 to $40 \mathrm{~T}$, consistent with the inset showing the converging (at $B_{c}=36 \mathrm{~T}$ ) of all the linear fittings from $\operatorname{Ln}\left[\left(2 e^{2} / h\right) \rho_{\max }\right]$ versus $B$ at different temperatures.

lar to the current direction (SI Fig. S6). This contrasts with the negative MR associated with chiral anomaly in 3D Dirac/Weyl semimetals 27] and with various scattering mechanisms [16, 28, as in those cases the negative MR disappears when the current is orthogonal to $B$. The field and temperature dependences we observed, as further discussed below, are also different from the behavior due to quantum interference effect in a variable-rangehopping regime [29].

We performed systematic $V_{t g}$ sweeps (fixed $V_{b g}=30$ $\mathrm{V})$ to extract $\rho_{\max }$ with temperatures at various inplane $B$ fields from $11.4 \mathrm{~T}$ to $45 \mathrm{~T}$ in Device N5. As shown in Fig. 3a, the insulating behavior of $\rho_{\max }(T)$ is strongly suppressed at higher fields. At the highest field $(45 \mathrm{~T}), \rho_{\max }$ saturates to a value close to $\sim h / 2 e^{2}$ and becomes relatively insensitive to temperature. We estimated the thermal activation gap $\Delta_{B}^{*}$ from the slope of $\operatorname{Ln}\left[\left(2 e^{2} / h\right) \rho_{\max }\right]$ versus $1 / T$ in the temperature range of $3 \mathrm{~K}$ to $22 \mathrm{~K}$ (Fig. 3a inset) and plotted it versus the corresponding $B$ in Fig. 3b, which also displays the gap size measured in another (second) cool down for $B$ up to $18 \mathrm{~T}$. The gap size $\Delta_{B}^{*}$ is found to differ slightly over different cool-downs but exhibits a similar dependence on $B$ in the intermediate field range $(5 \mathrm{~T} \sim 30 \mathrm{~T})$.

Extrapolating the linear fits in Fig. $3 \mathrm{~b}$ to zero suggests that the gap would close at a critical field $\left(B_{c}\right)$ between $36 \mathrm{~T}$ to $40 \mathrm{~T}$, around which we observe the sample (N5) to become metallic $\left(d \rho_{\max } / d T>0\right.$, see Fig. 3a) below $T \sim$ $2 \mathrm{~K}$. However, some non-metallic behavior $\left(d \rho_{\max } / d T<\right.$ 0 ) can still be observed between $2 \mathrm{~K}$ to $22 \mathrm{~K}$ even at the highest fields (Fig. 3a) and fitted to a thermal activation, giving data points that deviate from the red solid line (Fig. 3b). A non-metallic behavior under large in-plane magnetic fields was also observed in gapless samples such as $\mathrm{N} 2$ with $t=17 \mathrm{~nm}$ (SI Fig. S8b). The reason for this behavior remains to be understood. We have also verified that $\operatorname{Ln}\left[\left(2 e^{2} / h\right) \rho_{\max }\right]$ of sample N5 is linear with $B(<\sim 25 \mathrm{~T})$ at different temperatures and all the fitted 


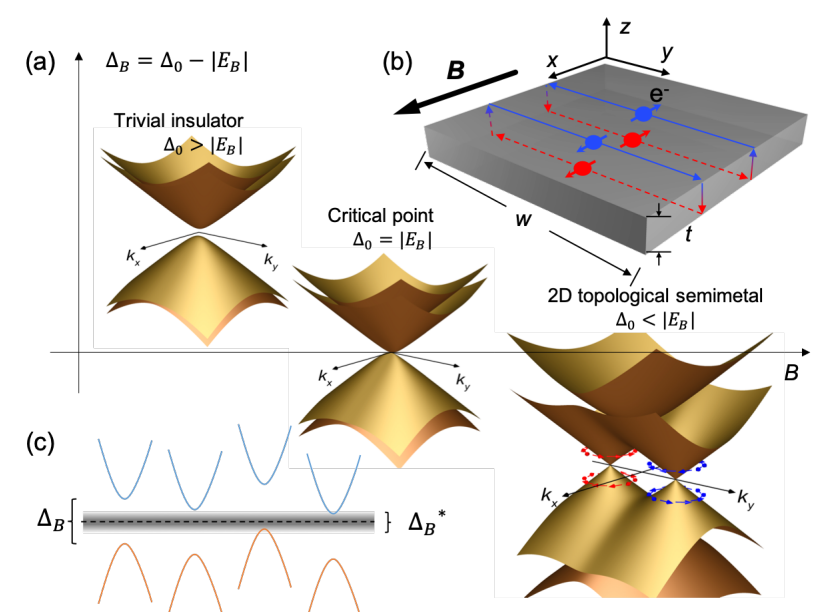

FIG. 4. (a) Predicted evolution of the surface band structure from a trivial insulator towards a 2D TSM, upon increasing inplane $B$ field in a thin TI sample with hybridized SS. Red/blue arrows depict spin textures of the gapless Dirac cones, separated in the momentum space for 2D TSM, respectively. (b) Schematic of the orbital motion (normal to $B$ and $x$ direction) of spin-helical SS electrons for a 3D TI film. (c) The gap size extracted from thermal activation can be underestimated (resulting in a smaller measured value $\Delta_{B}^{*}=\Delta_{B}-\delta$ ) compared to the real gap $\Delta_{B}$ due to the smearing effect of disorder induced potential fluctuations $(\delta)$ at different positions.

lines converge to a critical field of $\sim 36 \mathrm{~T}$ (inset of Fig. $3 \mathrm{~b})$. This also suggests $\Delta_{B}^{*} \propto\left(B_{c}-B\right)$, with a saturation resistivity $\sim h / 2 e^{2}$ (when $\Delta_{B}^{*} \sim 0$ ) and gap closing at $B_{c} \sim 36 \mathrm{~T}$ at fixed temperatures.

Such large negative $\mathrm{MR}$ and the corresponding insulator-semimetal transition have not been reported before in thin nonmagnetic 3D TIs with surface dominant conduction. Our observations of distinct behavior between thick and thin BSTS samples may be interpreted in terms of a theoretical prediction by A. A. Zyuzin et al. [15. Generally, in thick TIs the in-plane magnetic field $B$ (set to be along the $x$ direction) can introduce opposite shifts (along $k_{y}$ ) of top and bottom surface Dirac cones in the momentum space. This does not produce any MR effect in thick 3D TIs but will prevent the two DPs from annihilation and will tend to eliminate the hybridization gap in thin TIs (schematics shown in Fig. 4a). Semiclassically, a spin-helical SS electron with spin orientated along the $B$ field (thus spin magnetic moment $-g \mu_{B} / 2$, with $g$ being the in-plane spin g-factor and $\mu_{B}$ the Bohr magneton) moves clockwise around the circumference (Fig. 4b) with orbital magnetic moment (parallel to $B$ field) $\mu_{o r b}=\frac{e}{2(w+t) / v_{f}} w t \approx e t v_{f} / 2$ (noting width $w \gg t$ in our samples) [30. Both the spin and orbital magnetic moments couple to the $B$ field, giving rise to an effective Zeeman energy $E_{B}=g_{e f f} \mu_{B} B=\left(g \mu_{B}-e t v_{F}\right) B$ (total effective $g_{\text {eff }}=g-e t v_{F} / \mu_{B}$, the second term can also be considered as due to the Aharonov-Bohm phase gradient between the two opposite surfaces). In thin TIs with hybridization gap $\Delta_{0}$ (at zero $B$ field), one can show (Ref. [15. and SI) that the massive Dirac band is spin-split by the above "effective Zeeman energy", shrinking the hybridization gap linearly with $B$ as $\Delta_{B}=\Delta_{0}-\left|E_{B}\right|$. The gap vanishes at a critical field $B_{c}\left(\left|E_{B}\right|=\Delta_{0}\right)$, where the dispersion near $k=0$ becomes quadratic along $k_{y}$ and linear along $k_{x}$ (see Fig. 4a). With further increasing $B\left(>B_{c}\right)$, two DPs are restored and separated by $\sqrt{E_{B}^{2}-\Delta_{0}^{2}} / \hbar v_{f}$ along $k_{y}$. This gives rise to a distinct 2D TSM that is topologically stable as long as translational symmetry is preserved [15].

The above mechanism can qualitatively explain the trend we observed in experiments. However, the slope of the linear fitting yields the gap closing rate $\left|\frac{E_{B}}{B}\right| \approx 0.02$ $\mathrm{meV} / \mathrm{T}$ (corresponding to $g_{\text {eff }} \approx 0.33$ ). This is nearly two orders of magnitudes smaller compared with $\left|\frac{E_{B}}{B}\right| \approx$ $1 \mathrm{meV} / \mathrm{T}$ theoretically estimated for a $10 \mathrm{~nm}$ sample by A. A. Zyuzin et al. [15, which assumed $g=2$, leading to a negligibly small contribution from the Zeeman effect. Our results imply a large $g$, giving rise to a Zeeman term $\left(g \mu_{B} B\right)$ that is comparable with the orbital term $\left(e t v_{f} B\right)$. Thus, the two nearly cancel to give a small $\left|\frac{E_{B}}{B}\right|$. Assuming a typical $v_{f}=3.5 \times 10^{5} \mathrm{~m} / \mathrm{s}$ for topological SS with purely linear dispersion, we get a in-plane SS g-factor of $\sim 60$. In actual 3D TI materials such as BSTS, the surface Dirac cone contains substantial nonlinearity that can be described by a quadratic mass term added to the SS Hamiltonian. Subsequently, a reduced $v_{f} \approx 1.3 \times 10^{5} \mathrm{~m} / \mathrm{s}$, which describes the linear part in the Hamiltonian, yields a g-factor of $\sim 20$ (SI). It has been pointed out that the Zeeman coupling of the SS carriers can be highly anisotropic [31. In previous experiments, only an out-of-plane SS g-factor is determined and found to vary significantly in different TI materials [32, 33. Our study provides a method to extract the in-plane g-factor of SS carriers.

We have to note that in our experiments, the gap extracted from thermal activation is an effective transport gap $\left(\Delta_{B}^{*}\right)$ and can be smaller than the real band gap $\left(\Delta_{B}\right)$ due to disorder-induced smearing, namely $\Delta_{B}^{*}=\Delta_{B}-\delta$, where $\delta$ is a correction due to the potential fluctuations (likely to be on the order of several meV or higher 34]) in the system (Fig. 4c). Therefore, the observed apparent metallic behavior $\left(\Delta_{B}^{*}\right.$ reaching 0$)$ in Device N5 above $B_{c} \sim 36 \mathrm{~T}$ does not necessarily indicate the realization of the 2D TSM phase, which requires closing the real gap $\Delta_{B}$ and possibly much larger magnetic field than $B_{c}$ (noting the relatively small gap-closing rate of $0.02 \mathrm{meV} / \mathrm{T}$ in light of the estimated $\delta \sim \mathrm{meV}$ in our BSTS samples). It might be easier to realize the 2D TSM phase (at lower $B$ field) in other TI systems with a smaller or even negative g-factor (so the gap closing rate can be much larger than that in our samples). It would also be interesting for future studies to clarify whether the saturation resistivity $\sim h / 2 e^{2}$ is related to the modification of band structure and magnetic field induced spin-flip scatterings [20].

To summarize, we have demonstrated in ultrathin BSTS films with hybridized and gapped surface states 
a transition from an insulator to semimetal induced by either increasing thickness or an in-plane magnetic field. The in-plane magnetic field can shrink the hybridization gap and give a large negative MR that may be exploited for applications. Sufficient in-plane magnetic field is predicted to drive the thin 3D TI with hybridization gap to a 2D TSM phase, which would have 2 single-fold Dirac cones separated in the momentum space and provide a $2 \mathrm{D}$ analogue of Weyl semimetal (even though Weyl fermions cannot be strictly defined in even spatial dimensions [4]). Such a TSM (with the momentum space displacement between Dirac cones tunable by the in-plane magnetic field) can possess interesting 1D edge states [1, 35], which are analogous to the Fermi arcs in 3D Weyl semimetals [4] and have signatures that future experiments (e.g. performed at even higher magnetic fields) can search as evidence for the TSMs.

We thank A. Suslov, T. Murphy, J-H. Park and Z. Lu for experimental assistance, and Y. Jiang, C. Liu and K. T. Law for discussions. This work has benefited from partial support from DARPA MESO program (Grant N66001-11-1-4107) and NSF (Grant DMR \#1410942 and EFMA \#1641101). G. Jiang and R. Biswas were supported by Purdue University startup funds. A portion of this work was performed at the National High Magnetic Field Laboratory, which is supported by NSF Cooperative Agreement No. DMR-1157490, the State of Florida, and the U.S. Department of Energy.

* yongchen@purdue.edu

[1] A. H. Castro Neto, F. Guinea, N. M. R. Peres, K. S. Novoselov, and A. K. Geim, Reviews of Modern Physics 81, 109 (2009).

[2] M. Z. Hasan and C. L. Kane, Reviews of Modern Physics 82, 3045 (2010)

[3] X.-L. Qi and S.-C. Zhang, Reviews of Modern Physics 83, 1057 (2011)

[4] N. P. Armitage, E. J. Mele, and A. Vishwanath, Reviews of Modern Physics 90, 015001 (2018).

[5] L. Lu, J. D. Joannopoulos, and M. Soljačić, Nature Photonics 8, 821 (2014).

[6] M. Bellec, U. Kuhl, G. Montambaux, and F. Mortessagne, Physical Review Letters 110, 033902 (2013).

[7] L. Tarruell, D. Greif, T. Uehlinger, G. Jotzu, and T. Esslinger, Nature 483, 302 (2012)

[8] T. Wehling, A. Black-Schaffer, and A. Balatsky, Advances in Physics 63, 1 (2014)

[9] Y. Hasegawa, R. Konno, H. Nakano, and M. Kohmoto, Physical Review B 74, 033413 (2006)

[10] S. S. Baik, K. S. Kim, Y. Yi, and H. J. Choi, Nano Letters 15, 7788 (2015)

[11] V. M. Pereira, A. H. Castro Neto, and N. M. R. Peres, Physical Review B 80, 045401 (2009).

[12] J. Feilhauer, W. Apel, and L. Schweitzer, Physical Review B 92, 245424 (2015).

[13] J. Kim, S. S. Baik, S. H. Ryu, Y. Sohn, S. Park, B.-G.
Park, J. Denlinger, Y. Yi, H. J. Choi, and K. S. Kim, Science 349, 723 (2015).

[14] Y. Zhang, K. He, C.-Z. Chang, C.-L. Song, L.-L. Wang, X. Chen, J.-F. Jia, Z. Fang, X. Dai, W.-Y. Shan, S.-Q. Shen, Q. Niu, X.-L. Qi, S.-C. Zhang, X.-C. Ma, and Q.-K. Xue, Nature Physics 6, 584 (2010).

[15] A. A. Zyuzin, M. D. Hook, and A. A. Burkov, Physical Review B 83, 245428 (2011).

[16] S. Wiedmann, A. Jost, B. Fauqué, J. van Dijk, M. J. Meijer, T. Khouri, S. Pezzini, S. Grauer, S. Schreyeck, C. Brüne, H. Buhmann, L. W. Molenkamp, and N. E. Hussey, Physical Review B 94, 081302(R) (2016)

[17] O. Breunig, Z. Wang, A. A. Taskin, J. Lux, A. Rosch, and Y. Ando, Nature Communications 8, 15545 (2017), 1703.10784 .

[18] C. J. Lin, X. Y. He, J. Liao, X. X. Wang, V. Sacksteder IV, W. M. Yang, T. Guan, Q. M. Zhang, L. Gu, G. Y. Zhang, C. G. Zeng, X. Dai, K. H. Wu, and Y. Q. Li, Physical Review B 88, 041307(R) (2013).

[19] J. Liao, Y. Ou, X. Feng, S. Yang, C. Lin, W. Yang, K. Wu, K. He, X. Ma, Q. K. Xue, and Y. Li, Physical Review Letters 114, 216601 (2015).

[20] A. A. Taskin, H. F. Legg, F. Yang, S. Sasaki, Y. Kanai, K. Matsumoto, A. Rosch, and Y. Ando, Nature Communications 8, 1340 (2017)

[21] Y. Xu, I. Miotkowski, C. Liu, J. Tian, H. Nam, N. Alidoust, J. Hu, C.-K. Shih, M. Z. Hasan, and Y. P. Chen, Nature Physics 10, 956 (2014)

[22] Y. Xu, I. Miotkowski, and Y. P. Chen, Nature Communications 7, 11434 (2016).

[23] D. Kim, S. Cho, N. P. Butch, P. Syers, K. Kirshenbaum, S. Adam, J. Paglione, and M. S. Fuhrer, Nature Physics 8, $460(2012)$.

[24] V. Fatemi, B. Hunt, H. Steinberg, S. L. Eltinge, F. Mahmood, N. P. Butch, K. Watanabe, T. Taniguchi, N. Gedik, R. C. Ashoori, and P. Jarillo-Herrero, Physical Review Letters 113, 206801 (2014)

[25] S. Das Sarma and E. H. Hwang, Physical Review B 89, $235423(2014)$

[26] D. Kim, P. Syers, N. P. Butch, J. Paglione, and M. S. Fuhrer, Nature Communications 4, 2040 (2013).

[27] J. Xiong, S. K. Kushwaha, T. Liang, J. W. Krizan, M. Hirschberger, W. Wang, R. J. Cava, and N. P. Ong, Science 350, 413 (2015).

[28] P. Goswami, J. H. Pixley, and S. Das Sarma, Physical Review B 92, 075205 (2015).

[29] U. Sivan, O. Entin-Wohlman, and Y. Imry, Phys. Rev. Lett. 60, 1566 (1988).

[30] E. D. Minot, Y. Yaish, V. Sazonova, and P. L. McEuen, Nature 428, 536 (2004)

[31] R.-L. Chu, J. Shi, and S.-Q. Shen, Physical Review B 84, $085312(2011)$.

[32] A. A. Taskin and Y. Ando, Physical Review B 84, 035301 (2011)

[33] Y.-S. Fu, T. Hanaguri, K. Igarashi, M. Kawamura, M. S. Bahramy, and T. Sasagawa, Nature Communications 7, 10829 (2016)

[34] H. Nam, Y. Xu, I. Miotkowski, J. Tian, Y. P. Chen, C. Liu, M. Z. Hasan, W. Zhu, G. A. Fiete, and C.-K. Shih, Journal of Physics and Chemistry of Solids (2017), doi:10.1016/j.jpcs.2017.10.026

[35] R. Takahashi, in Topological States on Interfaces Protected by Symmetry (Springer Japan, Tokyo, 2015) pp. 63-71. 


\section{Supplementary Information}

\section{Effect of an in-plane magnetic field on the surface states of a 3D TI}

The basic theoretical model we use has been explained in ref. [1]. Here we present a detailed derivation and show how to understand the gap closing mechanism intuitively. Furthermore, we show how adding a quadratic term (not included in ref. [1]) to the Hamiltonian may affect the results.

The effective model of the Hamiltonian for the massless Dirac fermions of a 3D topological insulator surface state writes:

$$
H=\hbar v_{F}(\hat{z} \times \boldsymbol{\sigma}) \cdot \boldsymbol{k},
$$

where $\hbar$ is the reduced plank constant, $v_{F}$ is the Fermi velocity, $\hat{z}=(0,0,1)$ is the unit vector normal to the surface, $\boldsymbol{\sigma}=\left(\sigma_{x}, \sigma_{y}, \sigma_{z}\right)$ is the Pauli matrices (for the real spin) and $\boldsymbol{k}=$ $\left(k_{x}, k_{y}, k_{z}\right)$ is the wavevector. The corresponding gapless Dirac band $\left(\varepsilon= \pm \hbar v_{F}|\boldsymbol{k}|\right)$ for the surface states of a 3D TI film has a helical spin-momentum locking structure. As long as the film is thick enough and the time reversal symmetry is preserved, the two massless Dirac cones remain intact.

However, when a 3D TI film is thin enough, the tunneling (or so called hybridization) between the top and bottom surfaces must be considered. The thin TI system can be modeled by a surface state Hamiltonian which has the following form:

$$
H=\hbar v_{F} \tau_{z}(\hat{z} \times \boldsymbol{\sigma}) \cdot \boldsymbol{k}+\Delta \tau_{x},
$$

in the basis of $|u \uparrow>|, u \downarrow>, \mid d \uparrow>$ and $\mid d \downarrow>$, where the top and bottom surfaces can be considered as pseudospins ( $u$ and $d$, respectively) with corresponding Pauli matrices $\tau_{i}(i=\mathrm{x}, \mathrm{y}, \mathrm{z}), \uparrow$ and $\downarrow$ represent the real spin up and down states and $\Delta$ is the tunneling term in the zeroth order approximation (the identity matrix in the real spin space is implicit in the $\Delta \tau_{x}$ term). The band acquires a massive Dirac dispersion $\varepsilon= \pm \sqrt{\left(\hbar v_{F} \boldsymbol{k}\right)^{2}+\left(\Delta_{0} / 2\right)^{2}}$, where $\Delta_{0}=2 \Delta$ is the gap size.

Consider a thin 3D TI film with the top and bottom surfaces located at $z= \pm t / 2$, where $t$ is the film thickness. Assume the film is subject to a magnetic field $\boldsymbol{B}=B \hat{x}$ parallel to the film 
surface and in the $\mathrm{x}$ direction, we can introduce a vector potential $\boldsymbol{A}=-B z \hat{y}$ to the kinetic momentum $\hbar \boldsymbol{k}+e \boldsymbol{A}$. Now the TI surface state Hamiltonian becomes:

$$
H=v_{F} \tau_{z}(\hat{z} \times \boldsymbol{\sigma}) \cdot\left(\hbar \boldsymbol{k}-\frac{\tau_{z} e B t \hat{y}}{2}\right)+g \mu_{B} \sigma_{x} B / 2+\Delta \tau_{x},(3)
$$

where $g \mu_{B} \sigma_{x} B / 2$ is the Zeeman energy term with $g$ (which would be $\approx 2$ in free space) being the in-plane g-factor for the topological surface states and $\mu_{B}$ is the Bohr magneton. Since $\sigma_{x} B=$ $(\hat{z} \times \boldsymbol{\sigma}) \cdot B \hat{y}$, the Hamiltonian can be simplified as:

$$
H=v_{F} \tau_{z}(\hat{z} \times \boldsymbol{\sigma}) \cdot\left[\hbar \boldsymbol{k}-\tau_{z}\left(\frac{e t}{2}-\frac{g \mu_{B}}{2 v_{F}}\right) B \hat{y}\right]+\Delta \tau_{x},
$$

which corresponds to a $4 \times 4$ matrix:

$$
\left(\begin{array}{cccc}
0 & {\left[i k_{x}+\left(k_{y}-\kappa_{B}\right)\right] \hbar v_{F}} & \Delta & 0 \\
{\left[-i k_{x}+\left(k_{y}-\kappa_{B}\right)\right] \hbar v_{F}} & 0 & 0 & \Delta \\
\Delta & 0 & 0 & -\left[i k_{x}+\left(k_{y}+\kappa_{B}\right)\right] \hbar v_{F} \\
0 & \Delta & -\left[-i k_{x}+\left(k_{y}+\kappa_{B}\right)\right] \hbar v_{F} & 0
\end{array}\right)
$$

with a magnetic wavevector $\kappa_{B}=\left(\frac{e t}{2}-\frac{g \mu_{B}}{2 v_{F}}\right) B / \hbar$. The spectrum of the Hamiltonian is given by:

$$
\varepsilon= \pm \sqrt{\left(\hbar v_{F}\right)^{2}\left(k_{x}^{2}+k_{y}^{2}+\kappa_{B}^{2}\right)+\Delta^{2} \pm 2\left(\hbar v_{F} \kappa_{B}\right) \sqrt{\Delta^{2}+\left(\hbar v_{F} k_{y}\right)^{2}}}
$$

For non-zero $\Delta$, the surface band structure undergoes a quantum phase transition at a critical magnetic field $B=B_{c}$ satisfying $\left|\hbar v_{F} \kappa_{B}\right|=\Delta$. At $B<B_{c}$, the spectrum (shown in Fig. $4 \mathrm{a}$ and shown in Fig. S1a with two low-energy sub-bands attached with spin orientations) has solution $\varepsilon= \pm\left(\Delta \pm\left|\hbar v_{F} \kappa_{B}\right|\right)$ at $\boldsymbol{k}=0$. Here the band is trivial with a band gap of $\Delta_{B}=\Delta_{0}-\left|E_{B}\right|$, in which $E_{B}\left(=-2 \hbar v_{F} \kappa_{B}=\left(g \mu_{B}-e t v_{F}\right) B\right)$ defines an effective magnetic (Zeeman-like) energy. The two terms in $E_{B}$ correspond to the contributions from the actual Zeeman coupling and Aharonov-Bohm phase gradient, respectively. It can also be understood semiclassically as follows. For an electron moving around the circumference of a cross section (normal to $B$, see Fig. $4 \mathrm{~b}$ ) at velocity $v_{F}$, it gains extra energy from the magnetic field due to an orbital magnetic moment with magnitude of:

$$
\mu_{\text {orb }}=I * S=\frac{e}{2(w+t) / v_{F}} * w t=\frac{w t}{2(w+t)} e v_{F},
$$


where $I$ is the current generated by the electron surrounding the loop with area $S$ (rectangular shape with sides $w$ and $t$ ) and $e$ is the elementary charge. In the limit of $w>>t$, which is satisfied in our BSTS samples (usually with $w$ a few $\mu \mathrm{m}$ and $t$ tens of nm or less), $\mu_{\text {orb }}=e t v_{F} / 2$. Due to the spinmomentum locking of the surface states, the electrons with clockwise and counter-clockwise motion possess opposite spin directions that are parallel and anti-parallel to $\boldsymbol{B}$ (Fig. $4 \mathrm{~b}$ ), thus having opposite total magnetic moments of $\mp\left(\frac{g \mu_{B}}{2}-\frac{e t v_{F}}{2}\right)$, respectively. This gives a splitting (Fig. 4a) of each band (into two sub-bands) with the magnetic field, where one sub-band shifts up in energy by $\left|\left(g \mu_{B}-e t v_{F}\right) B / 2\right|$, and the other sub-band shifts down by the same amount. A similar analysis applies for the hole band near the gap. Overall, the gap size decreases by an "effective energy" (acting like a Zeeman energy) $E_{B}=\left(g \mu_{B}-e t v_{F}\right) B$. One can also define an effective gfactor $g_{e f f}=\frac{E_{B}}{B \mu_{B}}=\left(g-e t v_{F} / \mu_{B}\right)$ accordingly.

(a)

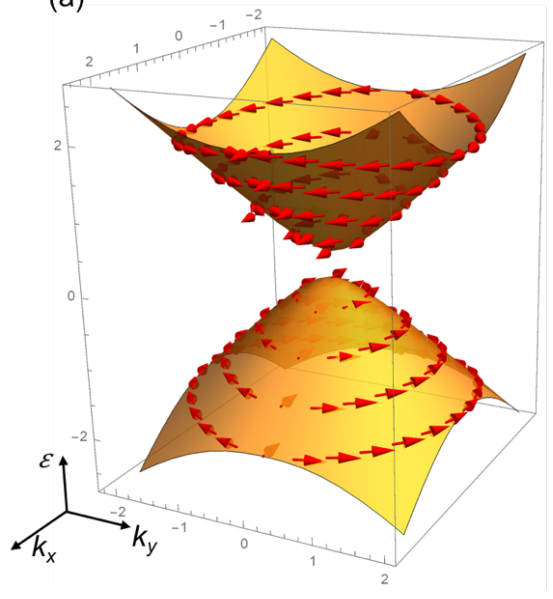

(b)

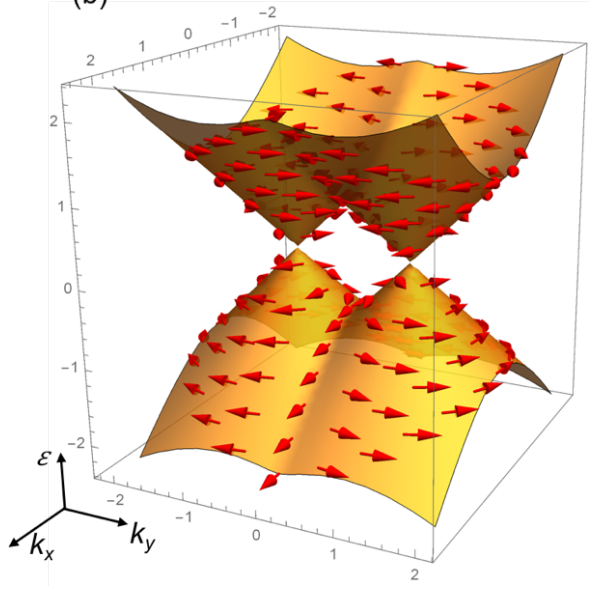

Fig. S1. (a) The two low-energy (with smallest $|\varepsilon|$ ) sub-bands from Equation (5) in the condition of $0<\mathrm{B}<\mathrm{Bc}$. The red arrows denote spin direction. (e-h) Similar plots for the $2 \mathrm{D}$ topological semimetal phase at $\mathrm{B}>\mathrm{Bc}$, where the two Dirac cones are recovered and have opposite spin helicity. The units for axis are arbitrary.

At $B>B_{c}$, the spectrum (shown in Fig. $2 \mathrm{G}$ and shown in Fig. S1b with two low-energy subbands attached with spin orientations) becomes gapless and restores two Dirac cones separated in the momentum space along the $k_{y}$ direction. This gives an intriguing topological 2D semimetal phase induced by the large in-plane magnetic field.

For $\Delta=0$, the band structure reduces to the case for thick films without hybridization: 


$$
\varepsilon= \pm \hbar v_{F} \sqrt{k_{x}^{2}+\left(k_{y} \pm \kappa_{B}\right)^{2}}
$$

which simply implies two relatively shifted Dirac cones in momentum space at $k_{y}= \pm \kappa_{B}$.

We have also considered a more realistic band structure with a quadratic term $\frac{(\hbar k)^{2}}{2 m^{*}}$ added to the Hamiltonian, with $m^{*}$ being the effective mass. It's found that only the Fermi velocity $v_{F}$ to the linear term $\hbar v_{F} \tau_{z}(\hat{z} \times \boldsymbol{\sigma}) \cdot \boldsymbol{k}$ contributes to the effective energy $E_{B}$. We estimated $m^{*} \approx$ $0.17 \mathrm{~m}_{e}$ and $v_{F} \approx 1.3 \times 10^{5} \mathrm{~m} / \mathrm{s}$ from the angle-resolved photoemission spectroscopy measurements of bulk BSTS [2]. Based on that and the relatively small $\left|\frac{E_{B}}{B}\right|$ value extracted from experiment for Device N5, we estimate $g \approx \frac{e t v_{F}}{\mu_{B}} \approx 20$.

\section{Sample preparation and measurement}

TI single crystals BiSbTeSe 2 (BSTS) were grown by the vertical Bridgman technique [3]. The dual-gated BSTS devices were fabricated into Hall-bar structures on highly p-doped Si substrates (with $300 \mathrm{~nm}$-thick $\mathrm{SiO}_{2}$ coating) with hexagonal boron nitride (h-BN) flakes (tens of $\mathrm{nm}$ in thickness) transferred onto the devices as top-gate dielectrics. Similar fabrication procedures for the dual-gated BSTS devices have been described elsewhere [4].

Transport measurements are performed in a helium-4 variable temperature system (with base temperature down to $1.6 \mathrm{~K}$ ) or helium-3 systems equipped with magnetic fields up to $18 \mathrm{~T}, 31 \mathrm{~T}$ or $45 \mathrm{~T}$ (down to $0.3 \mathrm{~K}$ ). The magnetoresistance MR is defined as $(\rho(B)-\rho(0)) / \rho(0)$, where $\rho(B)$ is the B-dependent resistivity. For all the transport measurements, Keithley 2400 source meters are used to apply gate voltages $\left(V_{\mathrm{tg}}\right.$ and $\left.V_{\mathrm{bg}}\right)$. Depending on the maximum resistance $\left(R_{\max }\right)$ of a specific sample, we measure it in different ways for better accuracy. For the most insulating samples (like Device N4 with $\mathrm{t}=6 \mathrm{~nm}$ and $R_{\max }>>\mathrm{M} \Omega$ ), we use two-terminal measurements. A constant AC (applied by Stanford SR830 lock-in with frequency $<10 \mathrm{~Hz}$ ) or DC (applied by Keithley 2400 source meter) source-drain voltage $(V=100 \mathrm{mV}$ or $10 \mathrm{mV})$ is applied across the sample while the current $(I)$ is monitored to give conductance $(=I / V)$. No measurable conductance or any gate tunability has been observed even at room temperature for BSTS flakes below $\sim 5 \mathrm{~nm}$, 
so we didn't show any data for these flakes. For samples with $R_{\max }$ less than a few M $\Omega$ (usually $t \geq 8 \mathrm{~nm}$ ), conventional four-terminal measurements with Hall bar structures (Fig. 1A) are used to measure the resistance. Generally, a low-frequency $(<20 \mathrm{~Hz})$ AC excitation current of $100 \mathrm{nA}$ (when $\left.R_{\max }<\sim 100 \mathrm{k} \Omega\right), 1 \mathrm{nA}\left(\sim 100 \mathrm{k} \Omega<\mathrm{R}_{\max }<\sim 1 \mathrm{M} \Omega\right)$ or $0.2 \mathrm{nA}\left(\sim 1 \mathrm{M} \Omega<R_{\max }<\sim 10 \mathrm{M} \Omega\right)$, where the current is reduced for larger $R_{\max }$ to avoid self-heating effect, is applied by a SR830 lock-in amplifier (input impedance $10 \mathrm{M} \Omega$ ). If $\sim 100 \mathrm{k} \Omega<R_{\max }<\sim 10 \mathrm{M} \Omega$, an extra voltage preamplifier (model DL 1201) with input impedance of $100 \mathrm{M} \Omega$ is added to improve the accuracy of voltage detection.

III. More details of the zero-field transport measurements and discussion

We have performed temperature dependent measurements of multiple samples with various thicknesses. In Fig. S2a, we replotted four representative data sets from Fig. $2 \mathrm{E}$ as $\sigma_{\min }\left(=1 / \rho_{\max }\right)$ versus $1 / T$, where $\sigma_{\min }\left(\right.$ or $\left.\rho_{\max }\right)$ is reached when both the two surfaces are gated to Dirac point or charge neutrality point. For samples with $t>\sim 10 \mathrm{~nm}$ (e.g. $t=14 \mathrm{~nm}), \rho_{\max }(\mathrm{T})$ only shows metallic behavior $\left(\mathrm{d} \rho_{\max } / \mathrm{d} T>0\right)$ below $\sim 50 \mathrm{~K}$, indicating gapless surface states. The size of non-zero effective transport gap $\Delta_{0}{ }^{*}$ in thinner BSTS samples is estimated from thermal activation fit with $\rho_{\max }(T) \propto e^{\Delta_{0}{ }^{*} / 2 k_{B} T}$ or $\sigma_{\min }(T) \propto e^{-\Delta_{0}^{*} / 2 k_{B} T}$ in appropriate temperature ranges, beyond which other conduction mechanisms need to be considered to describe the temperature dependent conduction. For example, in samples with $\mathrm{t}=10 \mathrm{~nm}$ (Device $\mathrm{N} 3$ and N5) and $8 \mathrm{~nm}$, the thermal activation fit has been applied to the temperature range between $\sim 3 \mathrm{~K}$ to $\sim 30 \mathrm{~K}$, where an insulating behavior is observed, to extract $\Delta_{0}{ }^{*}$ (note that it could be smaller than the real hybridization gap $\Delta_{0}$ due to smearing effect of potential fluctuations at different positions). This temperature range is chosen for the following reasons. Usually below $3 \mathrm{~K}, \sigma_{\min }$ tends to deviate from a single thermal activation fit. As shown in Fig. S2b of a typical example N5, we can fit the temperature dependent data down to $0.3 \mathrm{~K}$ with an empirical formula $\sigma_{\min }=C_{1} e^{-T_{1} / T}+C_{2} e^{-\left(T_{2} / T\right)^{1 / 3}}$, which gives $\mathrm{T}_{1}=\sim 8 \mathrm{~K}$ and $\mathrm{T}_{2}=\sim 0.02 \mathrm{~K}$. The second term has the form of variable range hopping $(\mathrm{VRH})$ for $2 \mathrm{D}$ (we caution that other forms may be used that also give reasonable fits), suggesting multiple 
conduction mechanisms at lower temperatures. Above $\sim 30 \mathrm{~K}$, Dirac points usually shift and the peak become much broader in gate-voltage sweeps (see Fig. S4a as a typical example), indicating additional conducting channels such as impurities or bulk states. In Fig. 4, a similar temperature range ( $3 \mathrm{~K} \sim 25 \mathrm{~K}$ ) has been used to fit the data of Device N5 under in-plane magnetic fields.

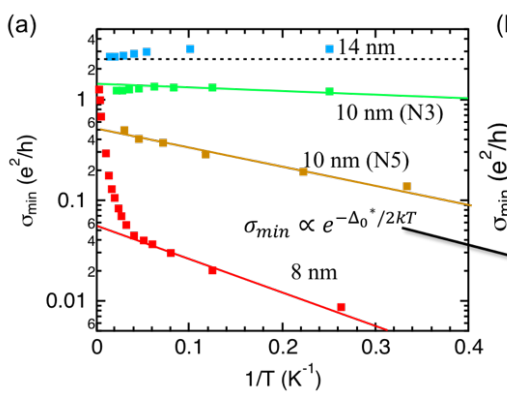

(b)

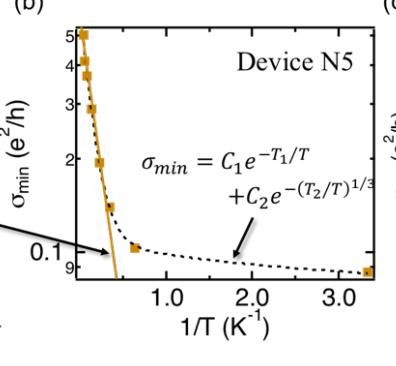

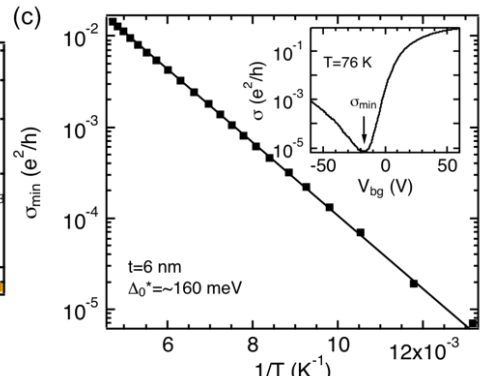

Fig. S2. (a) Same data from Fig. $1 \mathrm{~h}$ replotted as minimum conductivity $\sigma_{\min }\left(=1 / \rho_{\max }\right)$ versus $1 / \mathrm{T}$ for four BSTS samples. The solid lines are the corresponding fittings from thermal activation $\sigma_{\min }(T) \propto e^{-\Delta_{0}{ }^{*} / 2 k_{B} T}$ for the thin samples (typically in the temperature range between $3 \mathrm{~K}$ to $30 \mathrm{~K}$ ). The dashed horizontal line is a guide to the eye for a thicker $(14 \mathrm{~nm})$ sample showing an opposite trend (in $\sigma_{\min }$ versus $1 / \mathrm{T}$ ) compared to the thin ones, indicating a metallic behavior and a zero gap. (b) The $\sigma_{\min }$ versus $1 / \mathrm{T}$ for Device N5 (10 $\mathrm{nm}$ thick) in a larger temperature range (down to $0.3 \mathrm{~K}$ ). The yellow solid line is the same thermal activation fit in (a) for higher temperatures while the dashed curve is a fit based on the formula shown in the (b) for the whole temperature range. (c) In a 6-nm-thick sample, thermal activation fits well in the entire temperature range ( $76 \mathrm{~K}$ to $300 \mathrm{~K}$ ), giving a gap size of $\sim 160 \mathrm{meV}$. Inset is a typical back gate dependence for the 2-termial conductivity $\sigma$ measured at $76 \mathrm{~K}$ for this device.

In Fig. S2c, we showed the thermal activation fit for another $6 \mathrm{~nm}$-thick device in the temperature range of $76 \mathrm{~K}$ to $\sim 300 \mathrm{~K}$, below which $\sigma_{\text {min }}$ becomes too small to be detected accurately due to limitation of our instruments. The good linearity of $\sigma_{\min }$ in logarithmic scale versus $1 / \mathrm{T}$ indicates a hard gap opening in this device and the fitting gives a gap size $\sim 160 \mathrm{meV}$, which is much larger than that determined by angle-resolved photoemission spectroscopy (ARPES) measurements on $6 \mathrm{~nm} \mathrm{Bi}_{2} \mathrm{Se}_{3}$ grown by molecular beam epitaxy (surface gap barely vanishes at $6 \mathrm{~nm}$ ) [5] and the theory prediction for $\mathrm{Bi}_{2} \mathrm{Se}_{3}$ (a few $\mathrm{meV}$ at $6 \mathrm{~nm}$ ) [6] [7]. A much enhanced gap size has also reported previously on exfoliated $\mathrm{Bi}_{2} \mathrm{Se}_{3}$ thin flakes in ref. [8]. Given the material difference and better isolated surface conduction, the gap size in our thin BSTS films grows monotonically with reducing thickness and roughly follow an exponential function $\Delta_{0}{ }^{*} \propto$ $e^{-0.7\left[\frac{t}{(n m)}\right]}$ (Fig. 2F inset). We don't have evidence of oscillatory behavior of $\Delta_{0}{ }^{*}$ versus $t$ and did not observe any signs of quantum spin Hall effect [6] [7] [9]. 
The typical mobility of our sample with thickness $\geq \sim 10 \mathrm{~nm}$ is a few thousand $\mathrm{cm}^{2} / \mathrm{Vs}$, which can be referred in our previous publications (3). For Device N4 with thickness $6 \mathrm{~nm}$, the mobility is reduced to less than $100 \mathrm{~cm}^{2} / \mathrm{Vs}$ for both electron and hole doped sides. The scattering time $\tau$ of the samples can be estimated by $\tau=\hbar \sigma\left(\frac{2 \pi}{n}\right)^{\frac{1}{2}} /\left(e^{2} v_{f}\right)$ from Drude model and employing the Einstein relation. The corresponding mean free path can be obtained by $l=\tau v_{f}$, which ranges from $10 \sim 40 \mathrm{~nm}$ for typical samples ( $\geq \sim 10 \mathrm{~nm}$ thick).

\section{Additional information for Device N5 (Fig. S3 to Fig. S6)}

As seen in Fig. S3c, Device N5 (see device optical images in Fig. S3a and S3b) shows maximum resistivity $\rho_{\max }$ near $\mathrm{V}_{\mathrm{bg}} \approx 30 \mathrm{~V}$ and $\mathrm{V}_{\mathrm{tg}} \approx 1 \mathrm{~V}$ (note that depending on gate sweep histories, the exact peak position could vary slightly) at zero field and base temperature $\mathrm{T}=0.3 \mathrm{~K}$.

(a)

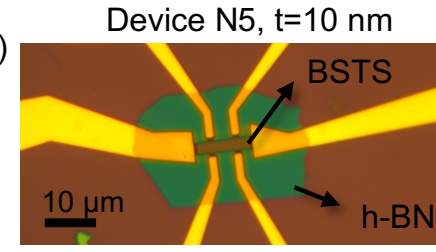

(b)

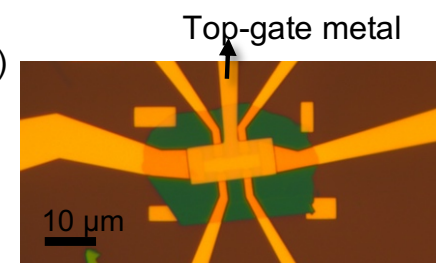

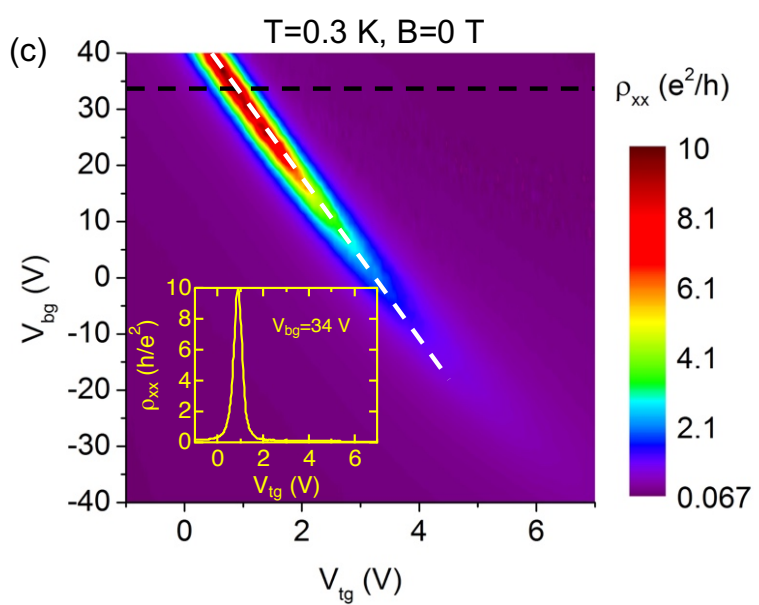

Fig. S3. (a,b) Optical images for the BSTS device N5 before and after top-gate metal deposition. The BSTS flake is etched into a Hall-bar structure and covered with a hexagonal boron nitride (h-BN) flake ( $24 \mathrm{~nm}$ thick) as top-gate dielectric. (c) The four-terminal $2 \mathrm{D}$ resistivity $\rho_{\mathrm{xx}}$ as a function of top and back gate voltages $\left(\mathrm{V}_{\mathrm{tg}}\right.$ and $\left.\mathrm{V}_{\mathrm{bg}}\right)$ for Device N5. The behavior is similar to that of Device N3 with resistivity peak along the total charge neutral line (dashed white line). However, Device N5 is initially more $\mathrm{n}$ doped and has a larger $\rho_{\max }\left(\sim 10 \mathrm{~h} / \mathrm{e}^{2}\right)$ as identified from the maximum resistivity peak in the line cut (shown in the inset) along the dashed line near $\mathrm{V}_{\mathrm{bg}}=34 \mathrm{~V}$. 
Most of the data of N5 shown in the main text were taken in a hybrid magnet system (up to 45 T) at NHMFL (Tallahassee, FL), referred as "first cool down". During the experiments, we notice that adjusting both gates is time consuming and could easily cause hysteresis thus uncertainties in the doping level of the two surfaces. Instead, for consecutive measurements (at different temperatures and magnetic fields), we fix $\mathrm{V}_{\mathrm{bg}}$ at $30 \mathrm{~V}$ and identify the resistivity peak in top gate $\left(\mathrm{V}_{\mathrm{tg}}\right)$ sweeps (always sweeping in the same range and same rate $\sim 40 \mathrm{sec} / \mathrm{V}$ ) as the global maximum resistivity $\rho_{\max }$ (shown in Fig. $2 \mathrm{f}$ and $3 \mathrm{a}$ ). We note that during the measurements of this set of data, the outer superconducting magnet of the hybrid magnet is maintained at $11.4 \mathrm{~T}$ and can not be changed. Fig. S4a and S4b show typical examples of these $V_{\operatorname{tg}}$ sweeps. In Fig. S4a the in-plane magnetic field is maintained at $11.4 \mathrm{~T}$ while the temperature is varied. In Fig. S4b the magnetic field is varied (using the inner resistive magnet of the hybrid magnet) while the temperature is kept at $0.3 \mathrm{~K}$. It confirms our general observation that neither the in-plane B field nor the temperature (below T 25 K) shifts the peak position (see also Fig. 2, Fig. S8 and Fig. S9). Therefore we can measure $\rho_{\max }$ at fixed gate voltages by continuously changing in-plane B fields in Fig. $2 \mathrm{f}$ (note the outside superconducting magnet is shut down during this specific run, allowing the sweeping of both positive and negative magnetic fields up to $\sim 34 \mathrm{~T}$ ) and Fig. S5 (measured at different temperatures below $\sim 25 \mathrm{~K}$ in a third cool down). The inset of Fig. S4a shows the typical configuration with in-plane field parallel to current direction for the measurements described in the main text and supplementary material. The only exceptions are Fig. S6 and Fig. S7 discussed below.
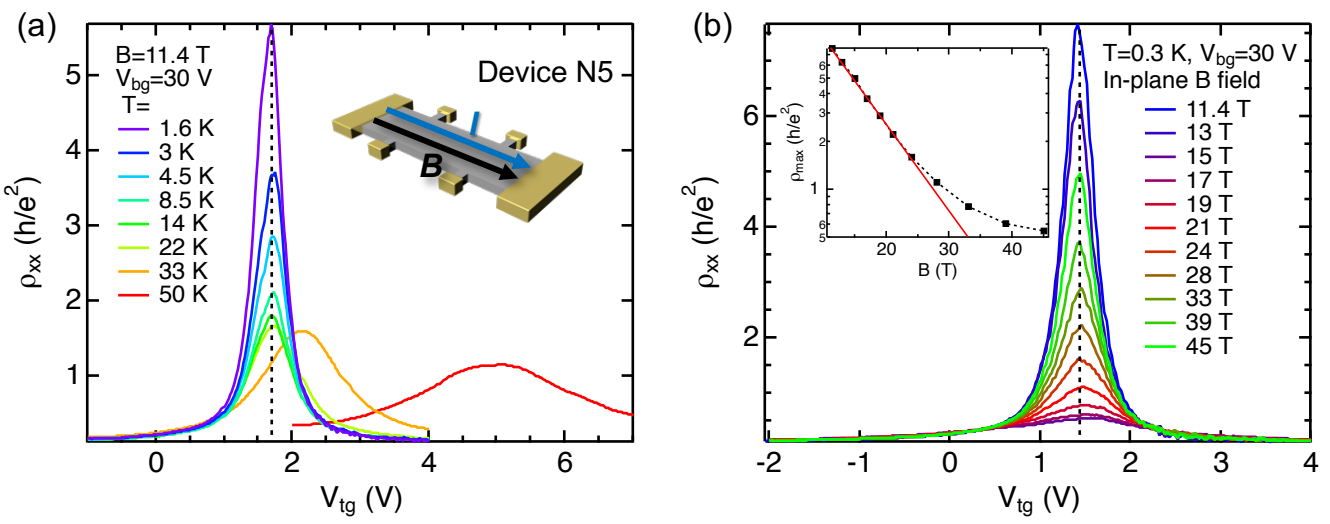

Fig. S4. The $\rho_{\mathrm{xx}}$ as a function of top gate voltage $\mathrm{V}_{\mathrm{tg}}$ with fixed $\mathrm{V}_{\mathrm{bg}}=30 \mathrm{~V}$ at (a) in-plane $\mathrm{B}=11.4 \mathrm{~T}$ and various temperatures and (b) (same data shown in Fig. 2e) different in-plane magnetic fields and $T=0.3 \mathrm{~K}$. 
Below $\sim 25 \mathrm{~K}$, the corresponding $\mathrm{V}_{\text {tg }}$ position of the resistivity peak is almost the same at $\sim 1.7 \mathrm{~V}$. Above $\sim 25 \mathrm{~K}$, the peak position deviates from the constant value $\sim 1.7 \mathrm{~V}$ and the peak value is not used for the thermal activation fittings in Fig. 4. The inset of (a) shows the measurement configuration with the in-plane field parallel to the current (I) direction. The inset of (b) shows the $\rho_{\max }$ (extracted from the peak values in b) in $\log$ scale as a function of the corresponding $B$ field. The red solid line is a guide to the eye of the linear dependence of $\log \left(\rho_{\max }\right)$ with B below $\sim 30 \mathrm{~T}$. Above $30 \mathrm{~T}, \rho_{\max }$ tends to saturate towards $\mathrm{h} / 2 \mathrm{e}^{2}$.

Fig. S5 shows an additional set of measurements (referred as the third cool down) of the dependence of $\rho_{\max }$ on the in-plane field $\mathrm{B}$ at different temperatures when both surfaces are tuned to charge neutrality points (fixed gate voltages). As shown in Fig. S5a, the low field features (below $5 \mathrm{~T}$ ), including a cusp near $\mathrm{B}=0$ and some positive $\mathrm{MR}$, are both smeared out at $\mathrm{T}>6 \mathrm{~K}$. The cusp feature was not observed in the less insulating sample N3, and could be attributed to quantum interference effect of VRH transport in a strong localization regime [10]. The positive MR may be related to the suppression of hopping transport due to spin polarization at localized states by moderate magnetic field $(\sim 5 \mathrm{~T})$ [11]. In contrast, the negative MR shown at higher fields $(>5 \mathrm{~T})$ is much more robust at elevated temperatures (still pronounced at $27 \mathrm{~K})$. The $\operatorname{Ln}\left[\left(2 \mathrm{e}^{2} / \mathrm{h}\right) \rho_{\max }\right]$ follows a linear dependence on $\mathrm{B}$ in the intermediate field range $(10 \mathrm{~T} \sim 30 \mathrm{~T})$, consistent with the plots in the inset of Fig. $3 \mathrm{~b}$ The $\rho_{\max }$ is observed to saturate toward $\sim \mathrm{h} / 2 \mathrm{e}^{2}$ at even higher fields (>30 T, inset of Fig. S4b).
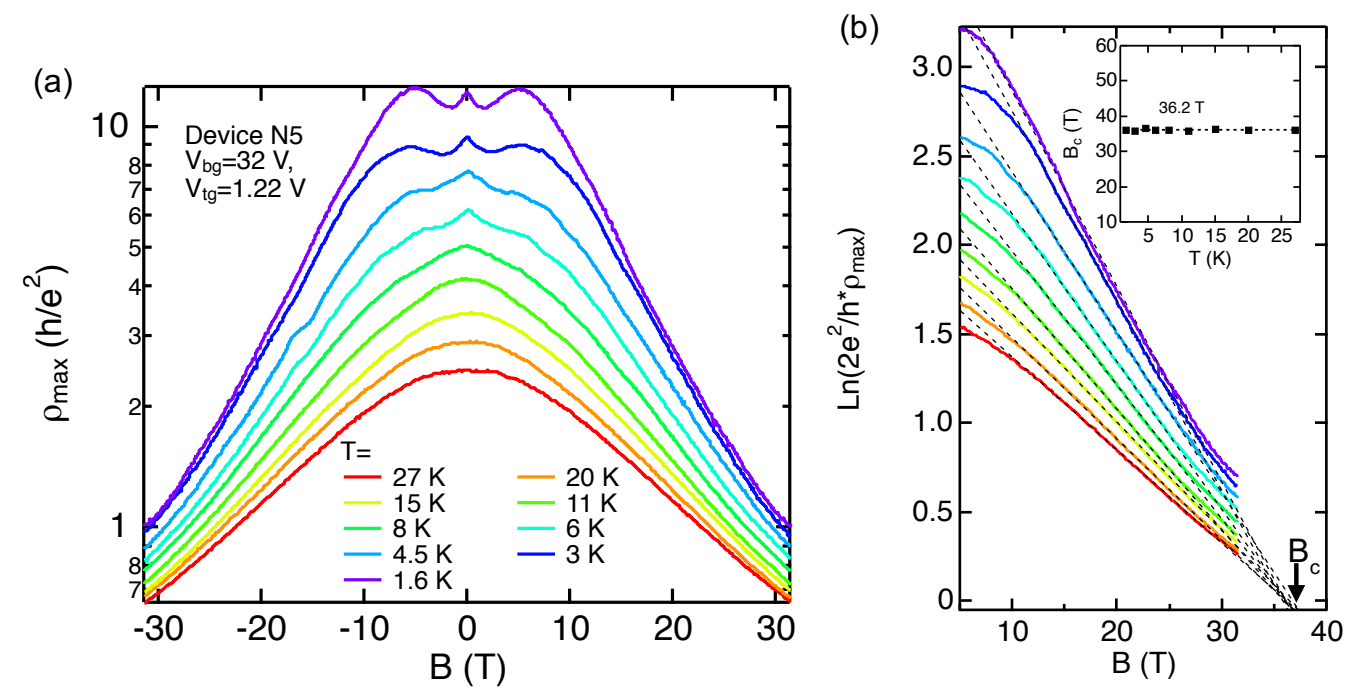

Fig. S5. (a) The measurements of $\rho_{\max }$ (at fixed gate voltages) in Device N5 as a function of the in-plane magnetic field B in different temperatures in a third cool down. The highest field is $31.5 \mathrm{~T}$. The $\rho_{\max }$ in $\log$ 
scale shows a linear dependence on B above a few T, with the corresponding plots of $\operatorname{Ln}\left[\left(2 \mathrm{e}^{2} / \mathrm{h}\right) \rho_{\max }\right]$ versus $\mathrm{B}$ in the positive field range shown in (b). All the linear fits (dashed lines) converge to a critical field $B_{c}=36.2 \mathrm{~T}$, with $B_{c}$ values for different curves extracted from the intercept on the B-axis plotted in the inset.

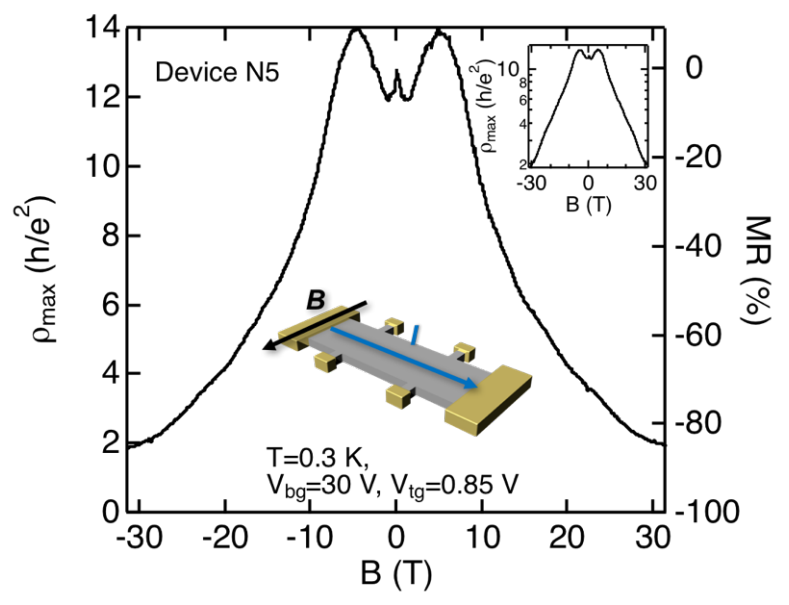

Fig. S6. The in-plane field dependence of $\rho_{\max }$ (left axis) and the corresponding MR (right axis) for Device $\mathrm{N} 5$ in a configuration (schematic in the inset) with in-plane field (B) orthogonal to the current (I). The top right inset shows $\rho_{\max }$ in $\log$ scale versus B.

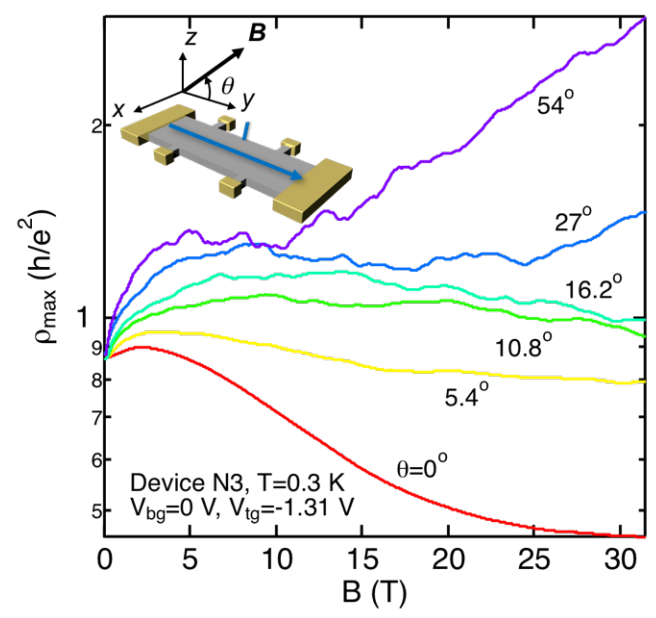

Fig. S7. The magnetic field dependence of $\rho_{\max }$ for Device N3 with various angles between the magnetic field (in the yz plane) and the sample surface (the xy plane). The y-axis is along the current direction and $\mathrm{z}$-axis is normal to the sample surface. The negative MR almost disappears with a small tilting angle $\theta \approx 10^{\circ}$, due to the out-of-plane component of the magnetic field.

According to the theory explained in part I and also in ref. [1], the negative MR has no requirement for the magnetic field direction as long as it is parallel to the surface (in-plane). In 
Fig. S6, we showed the magnetotransport data of Device N5 in a configuration where the in-plane magnetic field is perpendicular to the current direction. In this case, we still observe a large negative MR above $5 \mathrm{~T}$ and it reaches $-85 \%$ at $31 \mathrm{~T}$. The residual resistivity $\sim 2 \mathrm{~h} / \mathrm{e}^{2}$ at $31 \mathrm{~T}$ is found to be higher comparing to $\sim \mathrm{h} / \mathrm{e}^{2}$ in the parallel configuration (Fig. $2 \mathrm{f}$ ) where a rotation probe is used (same for all other in-plane field measurements) and allows fine adjustment of angles (according to the minima in $\mathrm{R}_{\mathrm{xx}}$ and $\left|\mathrm{R}_{\mathrm{xy}}\right|$ ) to minimize the out-of-plane component. Typically, outof-plane magnetic field induces positive MR (see an example of Device N3 in Fig. S7 while adjusting field direction relative to the sample plane). We note that the configuration used in this particular measurement (Fig. S6) could cause a small out-of-plane component of magnetic field due to imperfect alignment during sample mounting. This can partially explain the slightly higher resistivity at large fields comparing with the parallel-to-current configuration. More work may be needed to investigate if yet some other mechanisms can contribute to the small in-plane anisotropy.

\section{Additional Data from Thicker Samples}

In Fig. S8 and Fig. S9, we show more data on thicker $\left(>t_{c} \sim 10 \mathrm{~nm}\right)$ BSTS samples under inplane magnetic fields. In these samples, only positive MR is observed.
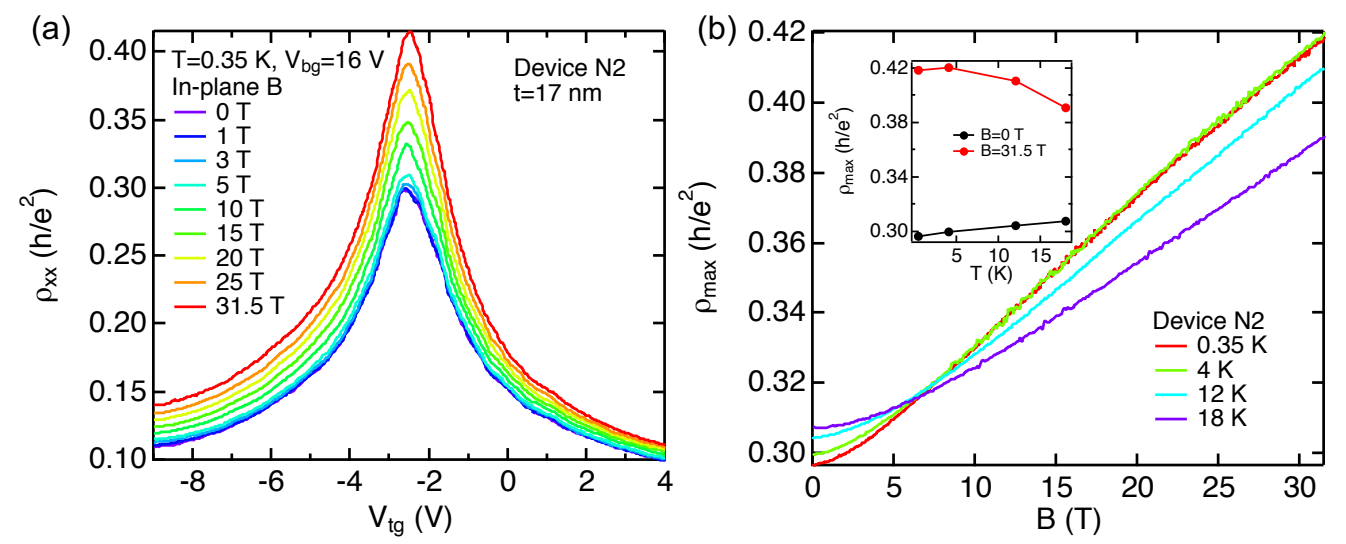

Fig. S8. (a) The raw data set for the 3D contour plot of Device N2 (17 nm thick gapless sample) in Fig. 3A, showing positive MR in the entire range of gate voltages. (b) The $\rho_{\max }$ as a function of in-plane field at different temperatures for Device N2. The MR generally follows a $\mathrm{B}^{2}$ dependence at low field and B dependence at higher field. With increasing B field, there is a crossover from the metallic to non-metallic 
behavior (seen from the temperature dependence of the data at fixed fields, with two examples at $0 \mathrm{~T}$ and $31.5 \mathrm{~T}$ shown in the inset) at $\sim 6 \mathrm{~T}$.
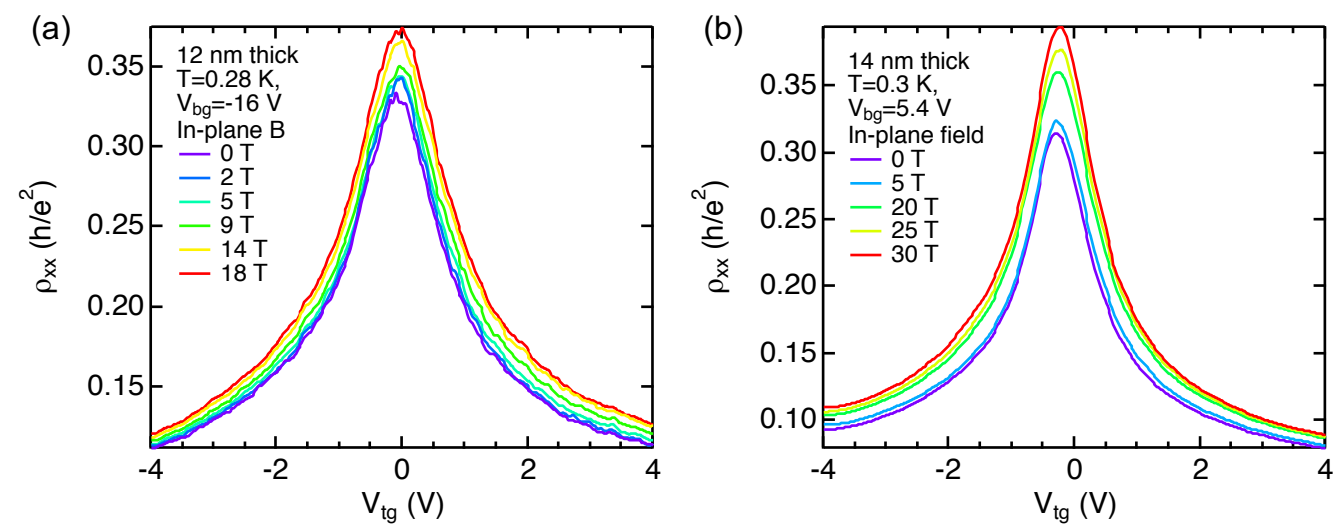

Fig. S9. Additional data showing the in-plane field effect on $\rho_{\mathrm{xx}}$ of another (a) $12 \mathrm{~nm}$ thick and (b) $14 \mathrm{~nm}$ thick sample, showing only small positive MR in the range of gate voltages. Both are measured by tuning the top gate voltage through $\rho_{\max }$ at fixed back gate voltages and base temperature $(0.3 \mathrm{~K})$.

\section{SI References:}

[1] A. A. Zyuzin, M. D. Hook, and A. A. Burkov, Phys. Rev. B 83, 245428 (2011).

[2] Chang Liu, private communications.

[3] Y. Xu, I. Miotkowski, C. Liu, J. Tian, H. Nam, N. Alidoust, J. Hu, C.-K. Shih, M. Z. Hasan, and Y. P. Chen, Nat. Phys. 10, 956 (2014).

[4] Y. Xu, I. Miotkowski, and Y. P. Chen, Nat. Commun. 7, 11434 (2016).

[5] Y. Zhang, K. He, C.-Z. Chang, C.-L. Song, L.-L. Wang, X. Chen, J.-F. Jia, Z. Fang, X. Dai, W.-Y. Shan, S.-Q. Shen, Q. Niu, X.-L. Qi, S.-C. Zhang, X.-C. Ma, and Q.-K. Xue, Nat. Phys. 6, 584 (2010).

[6] C.-X. Liu, H. Zhang, B. Yan, X.-L. Qi, T. Frauenheim, X. Dai, Z. Fang, and S.-C. Zhang, Phys. Rev. B 81, 041307 (2010).

[7] J. Linder, T. Yokoyama, and A. Sudbø, Phys. Rev. B 80, 205401 (2009).

[8] S. Cho, N. P. Butch, J. Paglione, and M. S. Fuhrer, Nano Lett. 11, 1925 (2011).

[9] H. Z. Lu, W. Y. Shan, W. Yao, Q. Niu, and S. Q. Shen, Phys. Rev. B - Condens. Matter Mater. Phys. 81, 115407 (2010).

[10] J. Liao, Y. Ou, X. Feng, S. Yang, C. Lin, W. Yang, K. Wu, K. He, X. Ma, Q. K. Xue, and Y. Li, Phys. Rev. Lett. 114, 216601 (2015). 
[11] I. Shlimak, E. Zion, A. V. Butenko, L. Wolfson, V. Richter, Y. Kaganovskii, A. Sharoni, A. Haran, D. Naveh, E. Kogan, and M. Kaveh, Phys. E Low-Dimensional Syst. Nanostructures 76, 158 (2016). 